\title{
André Ségal
}

Historien, retraité de l'enseignement au département d'Histoire

Université Laval

(1990)

\section{"L'éducation par l'histoire"}

Un document produit en version numérique par Jean-Marie Tremblay, bénévole, professeur de sociologie au Cégep de Chicoutimi

Courriel: jean-marie tremblay@uqac.ca

Site web pédagogique : http://www.uqac.ca/jmt-sociologue/

Dans le cadre de la collection: "Les classiques des sciences sociales"

Site web: http://www.uqac.ca/Classiques_des_sciences_sociales/

Une collection développée en collaboration avec la Bibliothèque

Paul-Émile-Boulet de l'Université du Québec à Chicoutimi

Site web: http://bibliotheque.uqac.ca/ 
Cette édition électronique a été réalisée par Jean-Marie Tremblay, bénévole, professeur de sociologie au Cégep de Chicoutimi à partir de l'article de :

\section{André Ségal, "L'éducation par l’histoire.”}

Un article publié dans le livre sous la direction de Fernand Dumont et Yves Martin, L'éducation 25 ans plus tard et après ?, pp. 241-266. Québec: l'Institut québécois de la recherche (IQRC), 1990, 432 pp.

M. André Ségal est historien, professeur retraité du département d'histoire de l’Université Laval depuis 1997.

[Autorisation formelle accordée par l'auteur de diffuser cet article le 15 novembre 2006 dans Les Classiques des sciences sociales.]

Courriel : Andre.Segal@hst.ulaval.ca

Polices de caractères utilisée :

Pour le texte: Times New Roman, 14 points.

Pour les citations : Times New Roman 12 points.

Pour les notes de bas de page : Times New Roman, 12 points.

Édition électronique réalisée avec le traitement de textes Microsoft Word 2004 pour Macintosh.

Mise en page sur papier format : LETTRE (US letter), 8.5" x 11")

Édition numérique réalisée le 6 décembre 2006 à Chicoutimi, Ville de Saguenay, province de Québec, Canada. 


\section{André Ségal}

historien, professeur retraité du département d'histoire depuis 1997

Université Laval

\section{"L'éducation par l'histoire" (1990)}

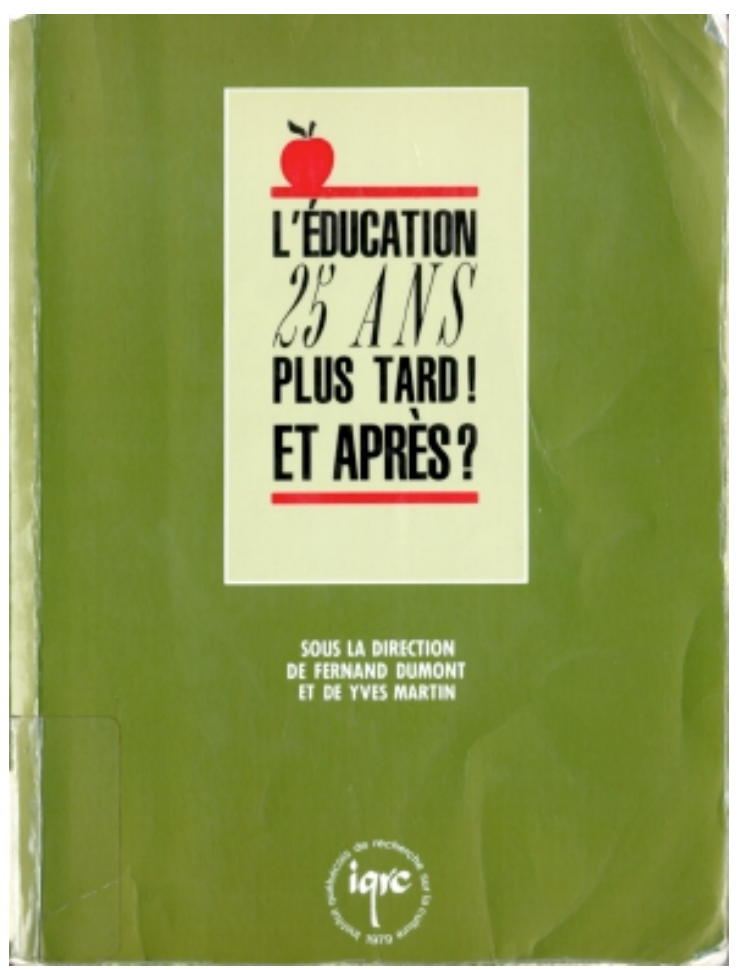

Un article publié dans le livre sous la direction de Fernand Dumont et Yves Martin, L'éducation 25 ans plus tard et après ?, pp. 241-266. Québec: l'Institut québécois de la recherche (IQRC), 1990, 432 pp. 


\section{Table des matières}

\section{Introduction}

Histoire, mémoire, éducation

Les fonctions éducatives

L'identité : les racines

L'altérité : la critique

Les pratiques éducatives

L'éducation permanente 
André Ségal,

"L'éducation par l'histoire"

Un article publié dans le livre sous la direction de Fernand Dumont et Yves Martin, L'éducation 25 ans plus tard et après ?, pp. 241-266. Québec: l'Institut québécois de la recherche (IQRC), 1990, 432 pp.

\section{Introduction}

\section{$\underline{\text { Retour à la table des matières }}$}

Nous proposons ici une réflexion sur la fonction de l'éducation historienne dans la culture des Québécois et particulièrement sur le rôle de l'école secondaire ${ }^{1}$. Nous ne tracerons pas l'évolution de l'enseignement de l'histoire depuis 1962, ni son bilan actuel. D'autres l'ont fait avec précision, optimisme ou ironie ${ }^{2}$. Par contre, nous tenterons de cerner quelques perspectives, dominées à vrai dire par le problème incontournable que posent les deux fonctions conférées à l'histoire et

1 Nous avons coutume d'employer « historien » comme adjectif pour distinguer l'activité présente, « recherche historienne », « production historienne », « éducation historienne » de son objet ou de ses matériaux passés, « source historique », « personnage historique », « changement historique ».

2 Le bilan le plus récent et le plus positif est celui de Micheline Dumont, « L'enseignement de l'histoire », Traces, no 27, avril 1989, pp. 29-36. L'évolution du dernier quart de siècle est tracée avec une verve décapante sous la forme du témoignage par Jean Breton, « Quand l'humble se raconte... », Traces, no 26, juillet 1988, pp. 14-21. Les moutures successives des programmes sont décrites par Louise Charpentier, "Qu'avons-nous enseigné ? », Bulletin de liaison S.P.H.Q., no 25, octobre 1987, pp. 31-36. D'une manière générale, le Bulletin de liaison de la Société des professeurs d'histoire du Québec, qui paraît depuis 1988 sous le titre de Traces, est un bon révélateur des genres et des niveaux des préoccupations et débats suscités parmi les enseignants d'histoire. 
pourtant peu compatibles : renforcer l'identité collective et développer l'esprit critique. La réflexion sur les fonctions éducatives de l'histoire nous amènera à nous interroger sur l'efficacité culturelle des pratiques éducatives.

L'enjeu est considérable, dans l'un et l'autre sens de la « culture ». La culture d'un groupe, son imaginaire, le système de référence commun qui aide à sa cohésion sociale sont fortement tributaires du rapport qu'il entretient avec le passé, sous forme de mémoire ou d'histoire $^{3}$. La culture personnelle, que j'aime définir comme « aptitude de la personne à dépasser son expérience propre et son environnement immédiat ${ }^{4}$ ", se bâtit fondamentalement par le contact avec les sociétés d'autres temps et d'autres lieux. Dans l'un et l'autre cas, l'enseignement scolaire de l'histoire pose les premières pierres et dessine les premières inclinations.

Avant d'aborder fonctions et pratiques éducatives, il nous paraît utile de répondre à deux questions préalables. L'une est factuelle : rappeler brièvement quelle place occupe actuellement l'histoire dans les programmes scolaires. L'autre est théorique : esquisser le cadre conceptuel dans lequel se situent nos réflexions.

La place de l'histoire est modeste dans la scolarité des adolescents. Elle est obligatoire en deuxième secondaire, sous le titre d'« Histoire générale ", et en quatrième secondaire, sous le titre d'« Histoire du Québec et du Canada », selon les programmes réformés en 1982 par le

3 Dans un article récent sur lequel nous reviendrons, Christian Laville fait le point sur l'importance du rapport au passé pour la cohérence des groupes sociaux («L'histoire et l'identité des minorités », Récits de vie et mémoires. Vers une anthropologie historique du souvenir, dir. par B. Jewsiewicki, [SainteFoy], Safi, [1988], pp. 147-188.

4 André Ségal, « Pour diffuser la culture historique », Recherches actuelles et mémoires collectives, Actes du CELAT, no 1, mars 1988, p. 49. Le lecteur nous permettra de ne pas noter chaque fois la référence quand, dans la suite du texte, nous emprunterons une idée ou une expression de cet article. 
ministère de l'Éducation ${ }^{5}$. Sur les cinq années du secondaire, l'histoire occupe obligatoirement en moyenne une heure et un tiers par semaine, ce qui est sensiblement plus que depuis 1977, mais sensiblement moins qu'avant ${ }^{6}$. Ces cours d'histoire sont précédés, en première et troisième secondaire, respectivement parla « Géographie générale » et la " Géographie du Québec et du Canada », ils sont suivis, en cinquième secondaire, par l'« Initiation à la vie économique ». De plus, un nouveau cours optionnel s'offre en cinquième secondaire, « Le 20e siècle : histoire et civilisations ». Il répond à un besoin évident, mais on craint que, dans la grande cafétéria des cours, il ne recueille que peu d'adeptes. Au niveau primaire, sont prévues des activités d'éveil aux sciences dites « humaines ». Quant au niveau collégial, l'apprentissage de l'histoire y est optionnel et très peu suivi ; il ne le sera guère plus selon la grille qui entrera bientôt en vigueur ${ }^{7}$.

\section{HISTOIRE, MÉMOIRE, ÉDUCATION}

\section{$\underline{\text { Retour à la table des matières }}$}

Pour esquisser le cercle de référence dans lequel nous nous inscrivons, il suffira d'indiquer la conception que nous nous faisons de l'histoire, de la mémoire et de l'éducation.

5 Histoire générale. 2e secondaire. Formation générale, 67 p. et Histoire du Québec et du Canada. 4, secondaire. Formation générale et professionnelle, 67 p. Ces programmes d'études ont été publiés l'un et l'autre en avril 1982 par le Gouvernement du Québec. La publication de ces programmes a été suivie de la publication par le gouvernement de Guides pédagogiques volumineux. Ensuite, de 1984 à 1986, six éditeurs ont produit les onze manuels qui se disputent le marché. On en trouvera la liste bibliographique dans M. Dumont, loc. cit., p. 30. L'implantation de ces programmes, particulièrement le plus neuf, celui de 2e secondaire, a été lente et difficile.

6 D'après L. Charpentier, loc. cit., p. 36.

7 Direction générale de l'enseignement collégial, Projet de réforme du programme de sciences humaines, 1989. 
Il importe d'abord de distinguer « mémoire » et « histoire ». L'une peut se définir comme le rapport empirique, pour une grande part irrationnel et implicite, que la société entretient avec son passé, tandis que l'autre est l'entreprise rationnelle et explicite d'élucidation de ce rapport ${ }^{8}$. Quel que soit le degré de scientificité de la production historienne, celle-ci est rationalisée, elle résulte d'un travail savant dans un cadre institutionnel. Certes, l'histoire produite par les historiens est un imaginaire construit, mais cette construction obéit à des règles qui tendent vers l'objectivité, sinon comme adéquation du savoir à l'objet étudié, au moins comme objectivisation de la procédure suivie. La construction historienne, à travers bien des avatars, se matérialise dans les livres d'histoire qui rejoignent le grand public et les manuels qui pénètrent dans les écoles 9 .

C'est assez clair. Par contre la " mémoire collective » est un concept nécessaire à l'analyse, mais qui rend compte d'une réalité difficile a saisir, surtout quand on se demande comment elle se construit ${ }^{10}$. Un des aspects principaux de la question est d'ailleurs de savoir quel est le rôle de l'histoire dans cette construction, ce qui revient à poser le problème de l'éducation historique. Pour la clarté du discours, la distinction entre mémoire et histoire est donc indispensable, bien que, dans la pratique, la frontière entre elles soit poreuse. De même que l'histoire contribue à construire la mémoire, la pratique historienne s'enracine inévitablement dans la mémoire de la société dont elle émane.

8 A. Ségal, toc. cit., p. 47.

9 Nous n'oublions pas les films, séries télévisées et matériels audiovisuels. Toutefois, les historiens, encore gens du livre, se sont jusqu'ici peu impliqués dans ces formes de diffusion. il en résulte un hiatus profond entre ces productions populaires et la production scientifique.

10 Ce problème a fait l'objet de séminaires du CELAT dont les exposés ont été publiés : Jacques Mathieu, «Étude de la construction de la mémoire collective des Québécois au XXe siècle/présentation générale », dans Jacques Mathieu (dir.), Étude de la construction de la mémoire collective des Québécois au XXe siècle. Approches multidisciplinaires, Cahiers du CELAT, no 5, novembre 1986, p. 3-6. 
Cependant, en raison même de sa prétention scientifique, l'activité historienne tend à rompre avec ses racines. Elle est d'abord une composante de la culture savante. On sait d'ailleurs le rôle majeur qu'elle a conquis, depuis vingt ans, dans cette culture en France et dans les pays de langue française, au point que certains y voient la réussite d'une grande stratégie médiatique au service de l'impérialisme des historiens 11 . Il y aurait lieu de s'interroger sur le sens des succès de cette industrie culturelle de luxe, sur sa portée au Québec et sur son rapport avec la mémoire et l'éducation.

Pour mettre fin à ces observations préalables, il nous reste à préciser la portée que nous donnons à l'« éducation ». L'éducation n'est pas l'instruction. Elle n'est pas seulement la formation de l'esprit par l'acquisition de savoirs intellectuels. L'éducation englobe l'instruction mais tend à développer toutes les facultés par lesquelles l'éduqué s'adapte à l'environnement naturel et social et devient capable d'y jouer un rôle personnel et conscient. L'éducation est inévitablement transmission de la culture sociale et donc instrument de reproduction de la collectivité. Mais elle est aussi nécessairement et prioritairement construction de la culture personnelle, donc de l'autonomie de l'individu. Éduquer, c'est conduire hors de soi, hors de l'expérience immédiate, hors de ce que certains nomment avec délectation le " vécu » personnel. Éduquer, c'est précisément ouvrir à ce que l'on n'a pas vécu soi-même, à un savoir extérieur, celui dont on hérite parce qu'il vient des générations précédentes, celui qu'on emprunte parce qu'il vient d'un monde étranger, parfois tout proche comme la classe sociale en haut de la pente douce, parfois lointain comme les pays du Soleil levant. L'éducation est bien entendu plus ou moins large, plus ou moins ouverte. Mais, dans tous les cas, elle s'oppose à l'« inducation », qui consisterait à ressasser le vécu personnel de l'« induqué », plutôt que de s'en servir comme d'un tremplin. À l'éducation ainsi conçue, l'histoire est admirablement adaptée.

C'est pourquoi le problème que nous avons à considérer dans les pages qui suivent est celui de la fonction de l'histoire dans l'activité éducative. Cette fonction est indissociable de celle de la mémoire. En

11 Par exemple, Guy Bourdé et Martin Hervé, Les écoles historiques, Paris, Seuil, (Points-Histoire), 1983, p. 201. 
effet, sans même aucune éducation historique, il se transmet des perceptions de la durée, des représentations du passé et des idées sur le changement social. Elles charrient habituellement les deux mythes contradictoires de l'âge d'or et du progrès. Dans cet univers de la rétrospection, l'éducation historique insère un effort de rationalité, de distanciation, de critique, d'objectivation. Cette éducation n'en véhicule pas moins des contenus mémoriels. Et - nous l'avons déjà suggéré - l'enracinement de l'histoire dans la mémoire engendre l'ambiguité fondamentale de la fonction éducative de l'histoire : identité ou altérité. Faut-il que l'éducation historique réponde au besoin d'identification ou développe la faculté de distanciation?

\section{LES FONCTIONS ÉDUCATIVES}

\section{$\underline{\text { Retour à la table des matières }}$}

L'histoire n'a guère d'application pratique. Les savoirs qu'elle produit ne sont guère utilisables ni dans les entreprises ni dans les administrations publiques, en dépit des avancées de la Public History ${ }^{12}$. Par contre, entreprises et administrations profitent de la qualité de formation de leurs cadres ; particulièrement quand ceux-ci ont acquis un certain sens de l'histoire, des conditions du changement, de la force du temps, et un ensemble de connaissances de type historien qui assurent et élargissent leur jugement professionnel. Les grands employeurs éprouvent de plus en plus le besoin d'un personnel doté d'une forte culture générale, plutôt que de spécialistes dont les techniques deviennent vite désuètes ${ }^{13}$. En ce sens, les fonctionnements économiques et politiques bénéficient de l'éducation historique, de la même manière que le travailleur, le consommateur, le citoyen.

12 Voir Barbara J. Howe et Emory L. Kemp, éd., Public History : an Introduction, Malabar, Fla., R.E. Krieget Pub. Co., 1966.

13 Par exemple Lynne Hall, Report Cardon Canadian MBA Programs, Queen's University School of Business, 1986, p. 5 et 10. 
En fait, la fonction sociale de l'histoire se réduit pratiquement à sa fonction éducative. Le savoir historien ne sert qu'à ceux qui le détiennent. Nul besoin d'avoir une éducation à la biologie pour profiter des applications de la biologie, par exemple, en prenant les médicaments que prescrit le médecin. Nul besoin de connaissances en génie mécanique pour profiter de ses applications, par exemple, en conduisant une voiture. Mais, il n'y a pas d'application de la connaissance historique dont on puisse profiter, si ce n'est en acquérant de la culture historienne.

Il fut un temps cependant où on croyait aux leçons de l'histoire. A cette époque, on recourait à l'expérience des anciens. Nous ne pensons pas ici à leur sagesse ou à leurs savoir-faire, mais à cette confiance qu'on avait dans la possibilité d'appliquer au présent des solutions qui avaient servi dans le passé ou d'éviter d'appliquer des recettes qui avaient autrefois failli. Comme si l'histoire se répétait, comme si elle n'était pas changement continuel, rendant sans cesse inadéquats les comportements naguère appropriés. En ce temps, l'histoire donnait des leçons et non seulement des leçons de morale religieuse, civique ou patriotique, mais aussi des leçons de politique.

Par une sorte de tradition rhétorique, certains tribuns ont continué jusqu'à de jour d'asséner les " leçons de l'histoire ». Dans ce genre, le débat référendaire fut une orgie, qui semble bien dépassée. Le discours que Pierre Bourgault tenait récemment, à l'ouverture du 26e Congrès de la Société des professeurs d'histoire, manifeste cette évolution. Il n'y est plus question de leçons du passé qui dicteraient les choix politiques d'aujourd'hui, mais de la nécessité d'une solide culture historique pour comprendre les données des problèmes et les différences entre les situations, les peuples, les cultures ${ }^{14}$.

Il n'est dès lors plus question d'applications pragmatiques de l'histoire suggérées, dans l'intérêt de tous, aux décideurs de la politique et de l'économie, mais bien de la nécessité pour chacun d'acquérir un savoir historien qui donne de la clairvoyance au comportement social. L'intérêt de l'histoire, son enjeu social et, partant, la première responsabilité sociale de la profession historienne réside désormais dans la

14 Pour en finir avec « l'histoire en cassettes », Traces, no 27, avril 1989, p. 9-13. 
fonction éducative. Sans doute n'est-ce pas la responsabilité première et directe de chaque historien. La fonction éducative repose sur une recherche rigoureuse, sans cesse approfondie et reprise à la lumière des connaissances et des problèmes nouveaux. Cette recherche requiert l'indépendance et la liberté d'esprit des chercheurs. Il faut donc que des historiens à la pointe de la recherche puissent se dégager des exigences de l'éducation historique. La profession dans son ensemble et la plupart de ses membres n'en ont pas moins à assumer cette responsabilité. Et ils ne le font guère 15 .

Or, si elle s'impose grossièrement avec assez d'évidence pour susciter des consensus distraits, la fonction éducative de l'histoire est, au Québec comme ailleurs, double et ambiguë. L'enseignant est déchiré entre les deux pôles. Selon la tradition la plus ancienne mais toujours très vivante dans le grand public, l'histoire doit fonder l'identité collective, attacher au passé, enraciner. Mais le public attend aussi de l'histoire, de plus en plus, et par son discours officiel surtout, qu'elle développe le sens critique. Est-il possible de remplir simultanément ces deux fonctions ? La fonction identitaire appelle le «nous »; elle tend à abolir les distances, elle conduit à penser que «nous avons été vaincus sur les Plaines d'Abraham ». La fonction critique appelle l'altérité. Elle invite à la distanciation dans le jugement. Elle constate que « l'armée, conduite par Montcalm, a été vaincue sur les Plaines d'Abraham ». Dans le premier cas, on s'interroge sur l'explication et la portée de ce drame ; dans le second, on s'interroge sur l'explication et la portée de ce fait.

Les historiens en général et particulièrement les didacticiens de l'histoire recommandent que l'éducation scolaire écarte la préoccupa-

15 Les signes de la distance excessive entre la pratique éducative et la pratique scientifique ne manquent pas. Par exemple, la Société des professeurs d'histoire du Québec regroupe les enseignants qui sont sur le front de l'éducation historique, mais les historiens des universités et des centres de recherche qui fréquentent cette société se comptent sur les doigts de deux mains. Ce sont souvent les mêmes et non pas nécessairement les plus représentatifs de la profession. 
tion identitaire au profit de la formation critique ${ }^{16}$. Henri Moniot analyse aussi le « double langage » de l'histoire, mais il le croit incontournable et « plutôt que de nier cette tension, de croire pouvoir en préserver le jeune âge ", il propose \& « entraîner progressivement les élèves à reconnaître pour ce qu'elles sont l'histoire critique et l'histoire intériorisée, à vivre consciemment leurs deux jeux, à saisir leurs exigences différentes et leur lien insécable 17 ». Il a certes raison de chasser l'illusion d'une éducation historienne purement critique. L'alternative qu'il indique me semble cependant quelque peu utopique, compte tenu des conditions d'enseignement et de la formation des maîtres. Il est peut-être possible par d'autres voies de rendre ces fonctions moins contradictoires. C'est ce que nous suggérerons plus loin.

Le discours officiel, celui des programmes, met aussi l'accent sur la fonction critique : "favoriser la réflexion, affiner le sens critique et développer l'objectivité 18 », « avoir acquis des concepts et des habiletés relatifs à l'étude de l'évolution des sociétés 19 ». De même, il insiste sur l'altérité, l'ouverture à l'autre, l'élargissement des horizons : « avoir développé une attitude d'ouverture et de respect à l'égard des valeurs autres que les siennes $20 »$. « faire preuve d'ouverture d'esprit à l'égard des valeurs étrangères à la société à laquelle il appartient 21 ». Et, quand les programmes proposent la fonction identitaire, ils le font avec modération. Il s'agit, par exemple, en histoire générale « de développer chez l'élève le sens de l'appartenance à une communauté élar-

16 C'est le cas de Christian Laville (loc. cit., pp. 177-181) et de Micheline Dumont (loc. cit., pp. 34-35), avec des justifications cependant différentes, plus idéologiques chez le premier, plus pragmatiques chez la seconde.

17 « Didactique de l'histoire », Dictionnaire des sciences historiques, dir. par André Burguière, Paris, PUF, [1986], p. 196. L'auteur exprimait déjà cette idée dans « Présentation », Enseigner l'histoire. Des manuels à la mémoire, Berne, Peter Lang, 1984, p. 122, no 1.

18 Histoire du Québec..., p. 11.

19 Histoire générale.... p. 13.

20 Histoire du Québec.... p. 13.

21 Histoire générale.... p. 13. 
gie 22 » ou en histoire du Québec et du Canada de « la compréhension de l'évolution de la société québécoise dans le contexte canadien, nord-américain et occidental 23 ». On précise que « l'histoire nationale [le mot est lâché] concerne tous les Québécois, quelle que soit leur origine ethnique, linguistique, sociale ou religieuse $24 »$. Suit l'invitation à refléter la diversité.

Que nous voilà loin de la célébration de « la civilisation française et catholique du Canada 25 » qui a prévalu jusqu'au rapport Parent. Ces programmes sont dans le droit fil de la politique scolaire énoncée en 1979 par lé gouvernement du Parti québécois où « L'école, foyer de la culture d'un peuple »est aussitôt précisé par « L'école, lieu ouvert $26 »$.

Le discours didactique et le discours officiel ne traduisent cependant pas toute la réalité ; loin s'en faut. Les attentes du public et la réponse des enseignants, la coloration de nombreux manuels et la structure même des programmes du secondaire contribuent encore à privilégier la fonction identitaire et le savoir mémorisé.

\section{L'IDENTITÉ : LES RACINES}

$\underline{\text { Retour à la table des matières }}$

Pour le plus large public, l'histoire est « histoire de... ». Non pas une démarche de raison, une méthode, une manière de savoir, mais un objet de savoir, un contenu, une démarche de mémorisation. Même en

22 lbid., p. 12.

23 Histoire du Québec.... p. 12.

24 lbid.

25 L. Charpentier, loc. cit., p. 31-32.

26 L'École québécoise, énoncé de politique et plan d'action, ministère de l'Éducation du Québec, Éditeur officiel du Québec, 1979, particulièrement pp. $16-17$. 
première année d'université, c'est un véritable labeur pédagogique que de faire élucider la polysémie du mot : histoire comme discipline, comme mode de savoir et Histoire comme Passé, comme objet de savoir. Le public attend qu'on lui raconte une histoire, son histoire. Il compte que l'enfant, l'adolescent apprenne « son histoire » à l'école, qu'il partage la mémoire des générations précédentes, qu'il s'alimente aux mêmes racines 27 . Les fruits en seront la fidélité au sentiment d'appartenance, le développement de l'identité collective. Dans tous les pays, le public associe l'histoire à l'identité collective, tantôt contre I'État assimilateur, tantôt avec l'État national ${ }^{28}$. Ce n'est pas moins vrai au Québec, bien que l'identité nationale y soit brouillée par la structure politique.

À ces attentes du public, les maîtres répondent positivement. Dans certains pays, ils y sont contraints, comme fonctionnaires, par les contrôles de l'État. Ailleurs, comme au Québec, les pratiques pédagogiques échappent largement à la surveillance des pouvoirs publics. Mais les maîtres y partagent, pour la plupart, les sentiments du public et se mettent volontiers au service de l'identité. Certes les héros identitaires ont varié selon le temps et les milieux : Saints martyrs, Patriotes, Pères de la Confédération ; ecclésiastiques, militants syndicaux, hauts fonctionnaires, entrepreneurs. Et l'évolution de ces héros fut particulièrement rapide et radicale depuis trente ans. Cette centration de l'enseignement de l'histoire sur le « nous » collectif est d'ailleurs soutenue par les manuels dont certains titres sont évocateurs : " À la recherche de mes racines », « Mon histoire », « Notre histoire » 29.

27 Cette version du plaidoyer pour l'histoire, note Henri Moniot (« Présentation p. 5), sert l'intérêt des adultes, tandis que l'autre poursuit l'intérêt des enfants.

28 Des exemples multiples et récents de ces rapports entre l'État, l'identité nationale et l'éducation historique ont été relevés par Marc Ferro, Comment on raconte l'histoire aux enfants à travers le monde entier, Paris, Payot, 1981 et L'histoire sous surveillance, Paris, Calmann-Lévy, 1986.

29 Gérard Cachat, Montréal, Lidec, 1984 ; François Charbonneau, Jacques Marchand et Jean-Pierre Sansregret, Montréal, Guérin, 1985 ; Danielle DionMcKinnon et Pierre Lalongé, Montréal, Éditions du renouveau pédagogique, 1984. 
En outre, la structure traditionnelle des programmes du secondaire contribue à privilégier la fonction identitaire. Histoire générale à treize ans en deuxième année, histoire nationale à quinze ans en quatrième année ${ }^{30}$. La progression pédagogique inviterait à l'inverse, du plus proche au plus éloigné, du plus connu au moins connu, du plus facile au plus difficile. Or, on mène d'abord l'enfant « De la préhistoire au siècle actuel 31 ». Il couvre des millénaires, la moitié da la planète et de multiples civilisations. Il n'applique pas ses premiers rudiments d'histoire aux sources voisines, aux témoignages accessibles, aux paysages familiers, mais à des mondes étranges et lointains. On ne s'étonne guère alors que le goût de l'exotisme l'emporte sur l'apprentissage de l'histoire ${ }^{32}$. Par contre, deux ans plus tard, quand l'adolescent a mûri, on lui propose l'étude d'une société unique, sur un espace et dans un temps réduits. Il n'y a pas là progression pédagogique, mais progression idéologique implicite du moins important au plus important. Ce qui importe le plus n'est pas l'acquisition progressive d'une méthode, ni la connaissance de l'évolution des civilisations dans la longue durée, mais bien l'histoire nationale, l'enracinement identitaire. La structure des programmes dément le discours des programmes.

Dans la perception des adolescents, le point de vue théorique des didacticiens, de qui venait sans doute la formulation des objectifs de programme, l'emporte-t-il sur l'opinion publique, à laquelle s'ajustent les enseignants et les contenus de programme ? On peut en douter. D'ailleurs, selon notre expérience, beaucoup d'étudiants qui s'inscrivent dans les programmes d'histoire des universités ont été préparés à y recevoir un savoir sûr et sécurisant.

Le type d'identification collective recherchée dans l'étude historique importe beaucoup. On a tort de percevoir a priori toute identité

30 Cette séquence du général au national n'a pas changé depuis 1962 (L. Charpentier, loc. cit., p. 34). On la retrouve aussi hors du Québec.

31 C'est le titre d'un des manuels : Guy Dauphinais, Montréal, Éditions du renouveau pédagogique, 1986.

32 Ainsi les pyramides d'Égypte ont un grand succès. Mais il faut éviter de confondre le goût de l'étrange et l'intérêt pour l'étranger, l'évasion vers ailleurs et la compréhension de l'autre. Il se peut qu'ils soient contradictoires. 
collective comme étroitement ethnique, nationale, voire nationalisante ou nationalitaire. Les groupes s'identifient par des réseaux complexes et hiérarchisés d'appartenance. Et si l'appartenance nationale occupait naguère, servie par l'historiographie, le champ central ou supérieur de l'identité, d'autres espaces culturels et d'autres valeurs suscitent aujourd'hui de larges adhésions. La fonction identitaire de l'histoire se joue aussi aux racines de ces espaces et de ces valeurs, par exemple, l'espace nord-américain ou la justice sociale.

Car l'identité des gens qui peuplent le territoire québécois n'est pas simple. Le peuplement est divers ; les appartenances, ambiguës ; les origines, lointaines. L'image de l'association simple d'un peuple et d'un territoire tient rarement. Au Québec, elle est particulièrement problématique. La réduction au plus petit dénominateur commun est donc tentante : le descendant des premiers colons, parlant encore français et vivant sur le soi provincial. Il serait excessivement optimiste de penser que ce schéma réducteur n'est que caricaturai et qu'il est partout dépassé. Il n'en est pas moins, presque partout, en voie de dépassement. Et ceci est important quant au rôle de l'éducation historienne et quant à la compatibilité de l'identitaire et du critique.

Considérons donc trois directions d'élargissement de l'identité collective et de l'éducation historienne : la multiplicité ethnique, le champ spatial, la profondeur temporelle.

Les Amérindiens sont au programme de quatrième secondaire. Dans le premier des sept modules, deux des six objectifs intermédiaires leur sont dédiés : " distinguer les influences réciproques des civilisations française et amérindiennes 33 ». Ce préambule à la NouvelleFrance ne signifie pas que le rapport inégal et actuel entre les cultures eurogènes et les cultures autochtones soit perçu comme constitutif de l'identité québécoise. Cela pourrait cependant advenir. De même l'identité du Québec contemporain comporte cette convivialité urbaine de communautés ethniques diverses formées par des strates d'immigrants dont les plus anciens forment la communauté majoritaire. Dans certaines écoles de Montréal, les enseignants réalisent à quelle crise aiguë peut conduire l'incurie à intégrer cette dernière dimension de no-

33 Histoire du Québec.... pp. 22-23. 
tre identité. Ailleurs, un autre enseignement de l'histoire peut encore devancer la crise. Il peut aider à comprendre comment l'identité d'un peuple change et comment une identité nouvelle s'articule sur l'identité précédente. L'intelligence du changement social n'est-elle pas le but même des études d'histoire?

Ainsi entendue, l'identité se borne encore à l'espace québécois. Mais qu'est-ce qui interdit de prendre en compte les autres appartenances qui parcourent ou englobent le champ psychosocial des Québécois et d'élargir ainsi le champ spatial de l'identité ? De l'appartenance canadienne - à nous les Rocheuses ! - je dirai peu, de crainte de révéler quelque parti pris. Les programmes scolaires y font la seule place sûre : institutionnelle. Le programme de quatrième secondaire s'intitule « Histoire du Québec et du Canada » suivant une des variantes sémantiques sur lesquelles les manuels jouent depuis le Vaugeois/ Lacoursière de 1968 qui s'intitulait bizarrement Canada-Québec : synthèse historique. L'appartenance à l'espace nord-américain est par contre négligée, alors que les solidarités des valeurs et des modes de vie la retraite en Floride - sont bien antérieures au Traité de libre-échange. L'appartenance à la francité n'est guère mieux servie, malgré la grande force identitaire de la langue. Elle est gênée par une francophobie latente héritée sans doute des méfiances de l'Église ultramontaine et des intérêts de l'Empire britannique. L'appartenance à la civilisation occidentale, l'identité occidentale des Québécois, est cependant l'orientation la plus prometteuse. Mais ici l'élargissement spatial est conditionnel à l'approfondissement temporel.

Les Québécois sont amputés de leur longue durée, bien plus peutêtre que les autres peuples d'Amérique. L'Américain de culture anglosaxonne connaît la rupture entre son histoire et celle de l'Europe. Il connaît aussi la continuité entre elles. Et l'histoire, sinon de l'Occident, au moins de l'Angleterre jusqu'au XVIe siècle fait partie du patrimoine américain et participe à son identité. C'est mieux que rien. Par contre, pour les Québécois, l'histoire identitaire commence brutalement avec Cartier ou Champlain. Comme si la Providence avait tout à coup créé une nouvelle culture pour l'implanter dans l'espace laurentien! Les siècles de gestation qui produisent dans l'Europe occidentale des structures mentales et religieuses originales, des coutumes et des savoir-faire spécifiques, des rapports sociaux et des régimes politiques 
particuliers qui sont importés dans l'espace américain, avant de s'y développer et de s'y transformer selon des dynamismes nouveaux, tout cela est ignoré. Et quand des programmes scolaires imposent, sous le nom barbare de " moyen âge ", l'étude des origines occidentales, celles-ci sont souvent traitées comme un monde étranger plutôt que comme le temps de l'élaboration de ce que nous sommes. C'est ainsi que, l'histoire de l'Église ne remontant jamais au-delà de la Réforme, catholiques et protestants n'ont pas de tronc commun, dans l'imaginaire québécois. Par contre, paradoxalement, legs de la culture intellectuelle et aristocratique des collèges classiques, les civilisations grecque et romaine de l'antiquité lointaine sont parfois présentées comme plus proches de nous. Bref, l'Atlantique, césure dans l'espace, est aussi césure dans le temps. Réintégrer dans le patrimoine québécois la longue durée de ses origines européennes, c'est sans doute la façon la plus féconde d'élargir l'identité québécoise et de consolider sa dimension occidentale ${ }^{34}$.

Il est illusoire de penser que l'enseignement de l'histoire et a fortiori la diffusion publique de l'histoire abandonne sa fonction identitaire. Le problème que nous avons posé est celui de la conciliation des deux fonctions. L'incompatibilité parait évidente quand la fonction identitaire conduit à rétrécir les champs d'étude, à stimuler les exclusions, à susciter des passions religieuses, patriotiques, nationalistes, à développer des oeillères. Nous avons montré cependant que l'identité collective pouvait n'être pas réduite à son plus petit commun dénominateur national. S'identifier comme Québécois, c'est s'identifier dans un rapport multi-ethnique complexe, c'est se définir par des appartenances particulières au Canada, à l'Amérique du Nord, à la Francité, c'est enfin surtout se reconnaître dans la longue durée occidentale.

Certes, ni le public, ni même les enseignants d'histoire ne vivent entièrement ainsi leur identification collective. Ces orientations ne

34 Mieux que ce faux nationalisme rétrospectif qui chercherait des origines soit dans les Îles britanniques, soit dans les pays qu'absorbera l'État français, l'approfondissement temporel ferait surgir comme bâtisseurs de notre présent aussi bien le moine bourguignon du XI. siècle, que le défricheur saxon du XII., que le tisserand flamand du XIlle, que le banquier florentin du XlVe, que le marin basque du XVe ou que l'imprimeur rhénan du XVIe. 
sont cependant pas utopiques; elles se dessinent lentement comme un fruit de la modernité québécoise post-référendaire. L'évolution des programmes, les pratiques scolaires et médiatiques, la recherche historienne peuvent contribuer à la maturation de ce fruit. Et, dans cette perspective, fonction critique et fonction identitaire de l'histoire deviennent peut-être conciliables ${ }^{35}$. L'enseignant peut échapper à la schizophrénie.

\section{L'ALTÉRITÉ : LA CRITIQUE}

\section{$\underline{\text { Retour à la table des matières }}$}

Il reste que c'est à la fonction critique de l'histoire que l'enseignant doit consacrer l'essentiel de ses énergies, pour la simple raison qu'il est seul à remplir ce rôle social. Les médias suscitent l'émotion et l'identification. Les médias véhiculent massivement l'information, non seulement sur tous les champs de la vie sociale présente, mais aussi, de plus en plus, sur le passé. Micheline Dumont décrit fort bien ce rapport inégal entre l'enseignant et les médias :

Ces fonctions de l'histoire, elles se produiront tôt ou tard via la politique, les médias, le marketing de telle ou telle idée, les bulletins de nouvelles, les émissions du Point ou de Caméra 89 et les sites historiques de Parcs Canada. Dans cet orchestre tonitruant, l'humble professeur d'histoire joue du piccolo. Il ne saurait rivaliser avec la force d'évocation d'un film, avec la puissance dramatique d'un bulletin de nouvelles, avec l'astuce d'une campagne publicitaire. Son rôle n'est pas de diffuser un message donné, il est justement avant tout de prépa-

35 Ce problème fait écho au débat beaucoup plus large et ancien entre la culture universelle et les cultures particulières. On en trouve une trace récente dans Alain Finkielkraut, La défaite de la pensée, Paris, Gallimard, (Folio/essai), 1987, 185 p. et dans la lecture critique qu'en fait Marcel Rioux, « Les frusques de la semaine et l'habit du dimanche », Possibles, vol. 12, été 1988, pp. 27-36. 
rer l'élève à affronter ce mitraillage d'informations disparates avec des instruments efficaces ${ }^{36}$.

Doter l'élève et ensuite l'étudiant de ces instruments efficaces, c'est enseigner l'histoire selon sa fonction critique. Ainsi l'histoire joue sa partition majeure dans la formation de la personne à l'autonomie et elle contribue, au-delà de l'école, à introduire « dans les mémoires collectives un sens critique qui, progressivement, démystifie le passé, le souvenir du passé et le discours sur le passé 37 ».

L'exercice de cette fonction critique dans l'éducation historienne, comment se manifeste-t-il ? Faute de guide systématique ${ }^{38}$, nous essaierons de répondre à cette question en partant du premier module des programmes du secondaire. Nous en marquerons la promesse et les faiblesses. Cela permettra d'illustrer quelques-uns des principaux objectifs d'apprentissage critique et de comprendre quels effets sociaux pourrait produire l'atteinte de ces objectifs.

Les objectifs généraux des cours sont centrés sur les apprentissages méthodiques : " avoir acquis des concepts et des habiletés relatifs à l'étude de l'évolution des sociétés 39 », " être initié à la démarche historique 40 », " avoir accru ses habiletés intellectuelles relatives à la démarche historique 41 », " être sensibilisé à la nécessité, aux princi-

36 Op. cit., p. 35.

37 André Ségal, « Mémoire collective et communication de l'histoire », Récits de vie et mémoires. Vers une anthropologie historique du souvenir, op. cit., p. 145 .

38 Bien que les revues pédagogiques fassent état de multiples expériences et que les didacticiens de l'histoire publient leurs réflexions, il n'existe pas de traité de didactique de l'histoire qui présente une synthèse des pratiques ou une théorie générale. Sans former non plus un ensemble systématique, sont très enrichissantes les onze études publiées par Christopher Portal (éd.), The History Curriculum for Teachers, Londres, The Falmer Press, 1987, 245 p.

39 Histoire générale..., p. 13.

40 Ibid.

41 Histoire du Québec.... p. 13. 
pes et aux limites de l'analyse historique des phénomènes sociaux 42 ». Cependant, sauf le premier, tous les modules des programmes d'histoire du secondaire sont déterminés par des contenus historiques disposés chronologiquement et les objectifs de ces modules sont orientés vers les apprentissages de ces contenus et non vers les apprentissages d'aptitudes historiennes. Comme nous l'avons déjà constaté sous un autre angle, il y a ici aussi divorce entre le discours du programme et la structure du programme. La structure invite à l'enseignement traditionnel, pour lequel il importe d'accumuler des connaissances factuelles, entre autres celles qui constituent les principaux repères identitaires.

Reste le premier module d'histoire générale. Son objectif ? « À la fin de ce module, l'élève devrait comprendre la nature de l'histoire et les moyens de s'initier à la connaissance historique 43 » et il est précisé que « ... les objectifs de ce premier module doivent être poursuivis tout au long du programme et ne peuvent être vraiment atteints qu'à la fin de ce dernier ${ }^{4} \gg$ ». Si cette précision n'était pas un voeu pieux, nous disposerions au Québec d'un programme exceptionnellement novateur. Chacun des deux volets du module illustre fort bien les types d'apprentissage qui seraient au coeur d'une histoire orientée vers sa fonction critique.

Dans le premier volet, il s'agit de « définir l'histoire comme étant l'étude de l'évolution des sociétés $45 »$. Cela nous introduit aux apprentissages conceptuels. Certes, les apprentissages factuels anciens recourent inévitablement à des termes abstraits : cause, conclusion, crise, croyance, économie, évolution, oligarchie, période, société, synthèse, travail... Mais ces termes restent des mots dont le sens est imprécis. Les premiers travaux des étudiants qui arrivent à l'université en té-

42 lbid.

43 Histoire générale.... p. 21.

44 Ibid.

45 lbid., p. 22. Nous avons fait l'analyse critique de cette unité dans « Enseigner la différence par l'histoire », Mélanges Van Santergen, Cahiers de Clio, no spécial, 1984, pp. 39-45. Les observations qui suivent sont inspirées de cet article. 
moignent de façon éclatante. L'apprentissage conceptuel au contraire vise systématiquement le maniement d'abstractions, non plus comme mots, mais comme outils de pensée. Dès lors la définition distraite et approximative ne suffit plus. Chaque concept exige un travail didactique avec explications et exercices pratiques répétés. On fait appel aux facultés d'abstraction toutes neuves de l'enfant et de l'adolescent et du même coup, loin de les laisser en friche, on les " cultive ", dans le sens le plus généreux du mot. Cela les médias ne peuvent le faire.

Le programme oriente cet apprentissage conceptuel dans trois directions : les concepts liés à la " société », les concepts liés à l'« évolution » et la " fonction de l'histoire ». Dans la première direction, il s'agit des diverses solidarités, familiale, territoriale, sociale et des catégories statistiques qui permettent de les analyser. L'élève apprendra entre autres la différence entre une " population », une " nation », un «État », ce qui apparaît bien comme un outillage conceptuel indispensable pour recevoir de manière critique les informations des médias et pour se comporter en citoyen conscient. En 1980, face au déferlement des propagandes référendaires, ce n'est pas un manque de connaissance sur les faits des histoires canadienne et québécoise qui a nui à la rationalité des choix, c'est le défaut d'outillage conceptuel («État», « nation »). L'éducation par l'histoire eût-elle été plus rationnelle dans les années antérieures que le résultat du référendum eût été peut-être différent.

La deuxième direction de l'apprentissage conceptuel concerne le temps. Au concept de base, l'« évolution » 46 , se rattachent tous les concepts du temps : présent, passé, futur ; rupture, continuité et rythmes du changement ${ }^{47}$. C'est un travail didactique essentiel mais ardu

46 Le terme d'« évolution » a une connotation positiviste et ethnocentrique : il renvoie à l'idée de " progrès ». Ce n'est pas un hasard : la structure générale des programmes suggère fortement une marche en avant continue de l'humanité, dont notre civilisation est l'aboutissement. C'est un des « changements » et plutôt que de définir l'histoire comme étude de l'évolution des sociétés, la définir comme étude du changement social.

47 À propos des rythmes du changement, voir André Ségal, « Pour une didactique de la durée », Enseigner l'histoire. Des manuels à la mémoire, op. cit., pp. 93-111. 
que de développer chez l'enfant et l'adolescent la conscience de ce que le changement n'est pas uniforme. Et quel meilleur usage faire du déroulement de l'Histoire? Il est mieux armé le citoyen qui aura compris que les mentalités ne changent pas nécessairement au rythme des techniques, ni les institutions au rythme des mentalités ${ }^{48}$.

La troisième direction est la réflexion sur la fonction de l'histoire. Elle est indispensable pour la raison pédagogique élémentaire que l'élève doit savoir à quoi sert ce qu'on lui fait apprendre. Elle est de plus indispensable à un second degré. Le citoyen baigne dans du discours historique et dans la mémoire collective. L'évocation du passé est partout présente, dans le langage quotidien, dans les discours idéologique, politique ou religieux, dans les séries télévisées. Pour négocier son rapport critique avec cet univers de la rétrospection, le citoyen doit avoir réfléchi sur la fonction sociale du rapport au passé. Où l'apprendra-t-il, sinon à l'école ? Et ce n'est pas facile à enseigner. Car il ne s'agit pas simplement de répondre à la question naïve et si naturelle : "À quoi ça sert l'histoire ? ». Il faut à chaque pas étudier le fait passé et le rapport de ce fait au présent. Ainsi, quand il est question de l'inévitable Christophe Colomb, après avoir présenté le fait avec toute l'illustration pédagogique qu'il permet, il faut aussi demander pourquoi la mémoire de ce personnage et de cet événement est si présente, pourquoi elle l'est tellement plus que les exploits des pêcheurs basques ou bretons dans les eaux glacées de l'Atlantique Nord.

Le projet de ce premier volet s'exprime en trois aphorismes : les sociétés sont différentes, l'évolution produit les différences, l'histoire explique ces différences. Avec le deuxième volet, on passe de la fonction de l'histoire au fonctionnement de l'histoire. Il s'agit d'« utiliser

48 Le programme introduit aussi les concepts de « performance » et de « relativité ». Ce sont des maladresses de la nouveauté, des plâtres à essuyer. La « relativité » est dans le jugement et non dans le temps, la « permanence » n'est pas un concept de l'histoire. Par rapport au temps historique, il y a des permanences physiques et même biologiques, mais parler de permanences historiques, c'est laisser croire qu'il existe des structures sociales immuables, en quelque sorte une nature sociale et non point seulement des cultures. Voir André Ségal, Enseigner la différence... 
des moyens qui permettent de retracer l'évolution des sociétés ${ }^{49}$ ». La façon la plus sûre de développer l'esprit critique n'est-elle pas d'initier à la méthode même de l'histoire 50 ?

Sans doute la recherche historique s'appuie sur une érudition considérable faite d'une grande somme de savoirs divers et de la maîtrise de techniques affinées. La mise en oeuvre de cette érudition n'en suit pas moins un schème intellectuel relativement simple. La pensée historique est proche de la pensée critique commune. Portant sur la matière sociale dans son ensemble et dans le temps, elle est la voie privilégiée de l'apprentissage de cette pensée ${ }^{51}$. "Penser, c'est savoir reconnaître les problèmes, distinguer les vrais des faux, en prendre la juste mesure, c'est savoir les résoudre en identifiant et en recueillant les informations pertinentes, en sachant traiter ces informations par l'analyse ; c'est savoir rassembler celles d'entre elles qui éclairent les problèmes afin de construire des explications ordonnées [... $]^{52} »$.

Le second volet du module « conceptuel » met en oeuvre l'apprentissage de la démarche en quatre étapes : la formulation de l'hypo-

49 Histoire générale, p. 23.

50 Près de nous, Christian Laville a orienté dans ce sens l'essentiel de ses recherches et de son enseignement de la didactique, par exemple : " Le manuel d'histoire : pour en finir avec la version de l'équipe gagnante », Enseigner l'histoire. Des manuels à la mémoire, op. cit., pp. 77-91 ; «Y a-t-il un didacticien dans la salle ?», Bulletin de liaison de la S.P.H.Q., no 23, avril 1985, pp. $11-18$; «L'histoire et l'identité des minorités », déjà cité ; « Le rôle de l'éducation historique », Traces, no 26, janvier 1988, pp. 33-35 ; Suzanne Citron (Enseigner l'histoire aujourd'hui. La mémoire perdue et retrouvée, Paris, Les Éditions ouvrières, 1984) propose plutôt une éducation à la mémoire.

51 Il est bien entendu que toutes les sciences sociales, à commencer parla géographie humaine, offrent les moyens de développer la pensée critique. Leurs méthodes sont cependant en général plus spécialisées, leurs concepts plus spécifiques et leurs champs plus étroits. C'est pourquoi, au risque d'être taxé d'impérialiste, nous maintenons que l'histoire a une vocation particulière dans la formation générale de l'esprit. Nous serions même porté à maintenir cette vocation jusqu'au niveau collégial.

52 Christian Laville, « Le rôle de l'éducation », op. cit., p. 34. 
thèse, la cueillette des informations, l'analyse des informations, la conclusion de l'enquête. Les manuels utilisent volontiers l'analogie avec l'enquête policière. C'est l'occasion de marquer une différence essentielle. Alors que l'enquête policière débouche sur un jugement moral, le jugement historique est d'une autre nature. Il ne recherche pas de coupable, ni n'établit des responsabilités. Il tente d'expliquer le pourquoi et le comment des changements sociaux. Cela aussi, c'est une tâche didactique qui requiert habileté, détermination et patience de la part du pédagogue. Faire comprendre que les changements sociaux résultent du jeu de forces sociales et non pas des bonnes ou mauvaises volontés de collectivités ou d'individus, c'est préparer le citoyen à sa maturité 53 . On admettra que cet objectif est infiniment plus fécond que celui de connaître l'oeuvre de Laurier ou de Périclès.

La deuxième unité du module s'attache aussi à la mesure du temps, ce que les programmes d'histoire font assez bien, depuis longtemps. Dépassant les techniques de datation (années, siècles, millénaires), elle apprend la périodisation et dépiste l'anachronisme. Sur ce point, les premiers jeux sont faciles. Qui ne s'amuserait du bracelet-montre au poignet du légionnaire romain ou de la bicyclette de Louis Hébert ? Tout autre est l'anachronisme mental, celui qui prête aux hommes des siècles précédents des valeurs, des motivations, des sentiments qui sont les nôtres. Ici plus qu'ailleurs s'apprend la distanciation, la connaissance de l'autre comme « autre » et non comme « même », la découverte de l'altérité, sans quoi ne se développent ni tolérance, ni respect, ni compréhension d'autrui. Et on mesure combien la fonction identitaire peut y nuire, du moins quand elle joue trop a l'étroit.

53 Une étude de Jocelyn Létourneau montre la distance à parcourir. Analysant les copies d'étudiants qui participaient au Concours Lionel-Groulx sur « La révolution tranquille : 1960-1968 », l'auteur montre à quel point le schéma mythique du bon et du mauvais, incarnés par Jean Lesage et Maurice Duplessis, l'emporte sur une analyse et un jugement qui seraient proprement historiens. Or, les auteurs de ces copies étaient choisis parmi les étudiants les mieux formés («L'imaginaire historique des jeunes québécois », Revue d'histoire de l'Amérique française, vol. 41, no 4, printemps 1988, pp. 553-573). Des aspects de cet article sont repris et abrégés sous le titre « La mémoire de la technocratie et l'impensable histoire du Québec », Traces, no 27, janvier 1989, pp. 2931). 
Enfin, cette unité a pour troisième objectif intermédiaire de « différencier certains types de documents ». Le recours aux documents n'est pas neuf dans l'enseignement de l'histoire. Il a autant suivi les progrès de la typographie que ceux de la pédagogie, avant même que ne triomphent les modes audiovisuelles. Mais en général le document (texte d'époque adapté, photographie de site, d'oeuvre d'art ou d'artefact) sert de support plus que de moyen didactique. Il illustre et souvent il distrait. Ici, cependant, il est traité et classé comme moyen même de la connaissance historique, comme témoignage ${ }^{54}$. L'observation de ces traces du passé et leur interprétation deviennent, le premier procédé d'apprentissage de l'histoire, tant pour la méthode historienne que pour l'objet historique. Reste à transférer l'opération mentale de l'élève au citoyen, de la trace du passé à l'objet d'actualité.

Ainsi donc, à écouter le discours didactique et les intentions programmatiques, à considérer le premier module du cours d'histoire générale, il semblerait que notre éducation scolaire fasse une belle place à la fonction critique de l'histoire. Certes, beaucoup de conditions sont favorables, plus sans doute que dans d'autres pays francophones. Mais les réalités demeurent assez loin des projets.

\section{LES PRATIQUES ÉDUCATIVES}

$\underline{\text { Retour à la table des matières }}$

Après avoir déclaré que les objectifs conceptuels du premier module doivent être poursuivis toute l'année, la structure des six modules suivants n'en tient guère compte et les meilleurs manuels à peine plus.

54 La classification suggérée par le programme est ambiguë. À preuve les interprétations données par certains manuels qui ne distinguent pas les traces du passé et la reproduction de ces traces. Voir notre note " Didactique et enseignement de l'histoire », Bulletin de liaison de la S.P.H.Q., vol. 23, no 2, janvier 1985, p. 19. Par ailleurs, certains manuels, cependant acceptés par le ministère et diffusés dans certaines écoles, manifestent une évidente régression quand ils substituent systématiquement aux reproductions de témoignages authentiques des textes imaginés par l'auteur et des reconstitutions dessinées par le maquettiste. 
Les enseignants restent dominés par la masse des enseignements factuels qu'on leur impose. Quant au programme de quatrième secondaire, on n'y trouve plus aucune trace des apprentissages proposés en deuxième. Les deux programmes s'ignorent. On sait cependant combien il est important que les apprentissages de savoir-faire soient entretenus et progressivement développés. Si de tels cloisonnements existent entre histoire générale et histoire nationale, que dire des cours de géographie et d'initiation à la vie économique ? Comment les cinq cours de sciences humaines convergeraient- ils vers un même développement intellectuel et social de l'élève ? Mais le succès d'une éducation historienne et critique et d'une véritable formation du citoyen autonome est encore bien plus compromis par les conditions mêmes de l'enseignement. Les pratiques éducatives historiennes sont mal servies par le système scolaire.

L'enseignant d'histoire au secondaire qui lit ces pages sourit doucement ou se met en colère. Selon son caractère, il se moque ou se révolte du bavardage universitaire. Il se soucie peu d'analyser les parts du conceptuel et du factuel dans ses pratiques, ni de savoir dans quelle mesure il oriente son enseignement vers le renforcement de l'identité ou l'ouverture à l'altérité. Il a le sentiment qu'il fait probablement tout cela en même temps selon les moyens du bord et les circonstances. Heureux quand il maintient la discipline et que ses élèves semblent intéressés. D'ailleurs, selon toute probabilité, cet enseignant a acquis sa formation en histoire a une époque ou ces problèmes ne se posaient pas, à moins qu'il n'ait jamais acquis de formation en histoire et que, mathématicien ou géographe, il enseigne l'histoire par la grâce administrative du bumping ${ }^{55}$.

Ni le climat des écoles, ni l'administration scolaire, ni l'état du corps enseignant ne favorisent la réorientation de l'éducation historienne vers le meilleur exercice de sa fonction sociale. Les succès dans ce sens sont l'oeuvre de marginaux. Sans doute le climat des écoles n'est favorable à aucune discipline. Il l'est encore moins dans les secteurs dont l'intérêt pragmatique n'est pas évident et que ne valorise pas l'opinion publique, surtout si l'effort intellectuel requis devient

55 Christian Laville (« Y a-t-il un didacticien ») a fait en 1985 une présentation, toujours valable, du rapport entre l'enseignant et le didacticien. 
comparable à celui qu'il parait normal d'attendre en mathématique, par exemple.

L'administration scolaire - à tous les niveaux - est peu sensible aux « petites matières ». Les enjeux sociaux de l'éducation ne la concernent pas. Seuls comptent le bon ordre et le budget. Les maîtres d'histoire sont des modèles; ils tiennent bien leurs classes et demandent peu d'investissements. La philosophie de l'éducation qui l'emporte est celle qui facilite la gestion. Nous illustrerons cette tendance par deux exemples.

L'administrateur donne la priorité à la pédagogie générale sur la didactique des disciplines. Il peut d'ailleurs avoir une certaine connaissance de la pédagogie, alors qu'il ne saurait connaître les didactiques de chacune des disciplines qu'il gère. Dans l'orientation du perfectionnement pédagogique, les problèmes généraux l'emportent presque toujours, particulièrement les questions d'évaluation. Cette philosophie aboutit à penser qu'un bon pédagogue peut enseigner n'importe quoi, s'il dispose du matériel adéquat ${ }^{56}$. Cela simplifie beaucoup la gestion du personnel. L'administrateur aurait raison dans une certaine mesure, si les apprentissages visés n'étaient que factuels : guidé par le livre du maître, un enseignant d'expérience peut expliquer le contenu d'un manuel. Il en va tout autrement quand il s'agit de transmettre les habiletés intellectuelles liées à la méthode d'une discipline. Il est exclu

56 Nous ne sommes pas hostile à la polyvalence des enseignants du secondaire, à condition que leur formation soit elle aussi polyvalente. Particulièrement, nous souhaiterions qu'existe un corps professoral unique pour les sciences humaines (géographie, économique, histoire) et préparé en ce sens par l'Université. Cela faciliterait la gestion du personnel - ce qui n'est pas un but - et surtout cela aiderait à la cohérence des enseignements et à la progression des apprentissages en sciences humaines d'une année à l'autre. Mais aussi longtemps que des mesures de formation n'auront pas produit leurs effets, les parachutages d'amateurs en histoire seront désastreux et de même dans d'autres disciplines. 
d'espérer le progrès d'une histoire méthodique, critique et conceptuelle, sans recourir à des maîtres formés dans la discipline 57 .

Cette polyvalence improvisée n'est pas plus souhaitable au niveau collégial. De là l'impuissance du pouvoir administratif à négocier avec les corporatismes disciplinaires. Le gouvernement a échoué dans sa tentative, pourtant très modeste, d'instituer un cours d'histoire obligatoire à ce niveau ${ }^{58}$. Quant aux cours de sciences humaines, ils se partagent en une dizaine de disciplines cloisonnées. Entre elles, aucune hiérarchie ! L'analyse du rôle spécifique de chacune d'elles dans la formation intellectuelle et sociale ainsi que dans la préparation aux études universitaires n'a pas été faite. C'est le triomphe de la cafétéria intellectuelle et, partant, des techniques de mise en marché des cours. Le problème n'est plus de bâtir les cours en fonction d'objectifs de formation mais en fonction des arguments de vente. Toutefois, espérons que la réforme qui entrera en application mettra de l'ordre dans l'offre des cours et réglementera quelque peu la libre concurrence ${ }^{59}$. Elle ne réglera, semble-t-il, aucun problème sur le fond puisqu'il n'appartient pas aux historiens de décider que l'histoire est une discipline de formation de base ${ }^{60}$. Encore eût-il fallu que le pouvoir administrateur pose la question et aménage les programmes du collégial, selon les finalités de celui-ci, quitte à prendre toutes les dispositions transitoires nécessaires au respect du personnel enseignant.

57 L'observation de Conrad Bureau (« Opinion », Contact, Québec, Université Laval, automne 1987, p. 31) s'applique aussi à l'enseignement de l'histoire : «En somme les futurs maitres n'apprennent plus le français : on leur apprend seulement à l'apprendre aux autres ».

58 Il aurait fallu réduire l'enseignement de la philosophie. Pourtant, dans notre expérience quotidienne d'enseignement en première année d'université, nous ne distinguons pas les traces qu'ont pu laisser quatre sessions de philosophie au cégep. Ces cours sont une survivance des traditions du collège classique et manifestent particulièrement bien la sclérose des programmes collégiaux.

59 Nous avons déjà fait référence à ce projet de réforme. Voir note 7.

60 À notre point de vue, l'histoire partage ce statut avec la géographie, la biologie, la psychologie et, bien entendu, les mathématiques. 
L'état général du corps enseignant n'est pas non plus actuellement un facteur de renouveau didactique. Certes, il s'en trouve chaque année pour animer leur société, organiser le congrès, produire le bulletin - et même en faire une revue. Il s'en trouve de Thetford à Baie-Comeau pour entreprendre de difficiles recyclages dans leur discipline, sans l'espoir d'aucune gratification matérielle. Il s'en trouve pour sacrifier plusieurs années de labeur ardu à la production d'un manuel. Nous saluons avec admiration ces hommes et ces femmes - une petite centaine - qui disposent de réserves d'énergie pour l'amour du métier. Il n'empêche que l'atmosphère dominante est à la fatigue ${ }^{61}$. Horaires excessifs, classes lourdes, problèmes de discipline, bureaucratie administrative, conflits de travail, dégradation du statut social, nous avons usé prématurément une génération entière d'hommes et de femmes, ceux-là mêmes à qui nous devons le formidable effort de démocratisation scolaire qui fait la fierté des Québécois.

Car il s'agit bien d'une génération d'un âge maintenant assez mûr. Ils sont grisonnants les pionniers des années soixante et soixante-dix. Parmi eux, faute de renouvellement, les jeunes diplômés sont rares ; ils font de la recherche, vivent de " jobines » ou ont quitté le domaine de l'histoire. Ces « anciens » connaissent bien leur métier et ont acquis depuis près de vingt ans leurs qualifications universitaires. Les connaissances alors acquises ont vieilli avec eux. Parfois elles se sont même rétrécies aux limites de leur enseignement quotidien. Leur savoir et celui qu'ils enseignent suivent mal les besoins d'une société en mutation rapide. Les conditions de travail gênent le perfectionnement de ceux qui souhaiteraient mettre à jour leurs problématiques historiennes et leurs connaissances historiques. Par ailleurs les administrateurs - nous l'avons dit - favorisent plutôt les perfectionnements pédagogiques en vase clos.

La tendance à élargir la fonction identitaire de l'éducation historique et à privilégier sa fonction critique est désormais manifeste et répond sans doute adéquatement aux besoins de la société québécoise. Toutefois cette évolution reste compromise en raison d'incohérences dans la structure des programmes, des blocages administratifs et du

61 Au sujet de l'état du corps enseignant, voir Christian Laville, « Y a-t-il un didacticien... », loc. cit. 
vieillissement du corps professoral. Les perspectives sont-elles donc décourageantes ? Dans le court terme, peut-être. À plus long terme cependant, l'école adaptera son éducation historienne à la nouvelle modernité du Québec, tout comme elle l'a fait après 1960 62. Cela dépendra essentiellement de trois facteurs : la volonté politique, le renouveau du corps enseignant et la réflexion programmatique.

Dans une conjoncture où les milieux d'affaires commencent à ressentir la pénurie d'un personnel cultivé, les pouvoirs publics ont commencé à s'émouvoir des lacunes dans l'apprentissage de la langue maternelle - ce qui était le plus urgent. Il est vraisemblable qu'ils se soucieront aussi bientôt de la formation intellectuelle et sociale, d'autant plus que l'histoire a cessé d'apparaître comme un instrument révolutionnaire.

Inévitablement, l'école recommencera bientôt à recruter des enseignants. Deux questions se poseront. Ces recrues seront-elles recherchées parmi les jeunes candidats les plus brillants et disposant de la meilleure formation en histoire ? Ces recrues seront-elles accueillies et stimulées à mettre en oeuvre leurs savoirs, leur enthousiasme et leur inexpérience ou devront-elles d'abord se ranger?

Les programmes sont réformés environ aux dix ans. La prochaine réforme interviendra probablement à la fin des années quatre-vingtdix. Sera-t-elle préparée par une réflexion systématique sur les fonctions sociales de la formation historienne et sur l'état actuel des connaissances en histoire comme en didactique ? Ou résultera-t-elle de négociations et de compromis entre enseignants, historiens, didacticiens et administrateurs?

62 Micheline Dumont (loc. cit.) montre très bien le virage pris dans les années soixante et poursuivi depuis. Elle en tire une conclusion fort optimiste quant à la santé actuelle de l'enseignement de l'histoire. Notre point de vue est différent en ce que nous pensons qu'il y a un nouveau virage à prendre et qu'il tarde. 


\section{L'ÉDUCATION PERMANENTE}

Retour à la table des matières

Quoi qu'il en soit, même si l'éducation historienne offerte à l'école était la mieux adaptée qui soit aux élèves et au présent, " les enfants ne pourraient à treize ou quinze ans acquérir une culture qui s'adapterait automatiquement à leur maturation et leur servirait pour la vie 63 ». L'éducation scolaire n'est que le début de l'éducation permanente. Or, l'histoire est omniprésente dans l'éducation permanente, sous mille formes : livres et périodiques, films et séries télévisées, jeux et théâtre, musées et centres d'interprétation. Là se trouve un autre enjeu de l'éducation historique et qui échappe à l'action institutionnelle.

Des orientations de l'éducation scolaire et de l'éducation permanente, les historiens, et les intellectuels en général, ne peuvent se désintéresser. L'éducation historique est une responsabilité des départements universitaires et de tous les centres de recherche voués à la culture. Leur implication sera un autre facteur du progrès de cette éducation, quand, désertant les tours d'ivoire, ils développeront leur attention critique et qu'ils apprendront à participer à la production des instruments d'éducation populaire ${ }^{64}$.

Car « la démarche historienne se révèle une des modalités de la recherche de l'Autre, par quoi se définit l'historicité de l'Homme, et son intention dernière, comme la reconstitution des multiples visages de la liberté 65 ».

63 André Ségal, « Pour diffuser la culture... », loc. cit., p. 50.

64 Dans un mémoire de maîtrise qu'il est occupé à terminer, Pascal Lapointe montre à quel point les historiens ont privé jusqu'ici les réalisations historiques télévisuelles de leur attention critique.

65 Nicole Gagnon et jean Hamelin, L'homme historien, St-Hyacinthe, Edisem, 1979 (Méthodes des sciences humaines), p. 118. 